Research Article

\title{
Contribution Rate Evaluation Method of Equipment System of Systems Based on Fault Tree
}

\author{
Min Du (D), Zhonghua Cheng (D), and Enzhi Dong (i) \\ Shijiazhuang Campus of Army Engineering University, Shijiazhuang 050003, China \\ Correspondence should be addressed to Min Du; 2120682856@qq.com
}

Received 28 May 2021; Accepted 2 September 2021; Published 15 September 2021

Academic Editor: Francesco Soldovieri

Copyright (c) $2021 \mathrm{Min}$ Du et al. This is an open access article distributed under the Creative Commons Attribution License, which permits unrestricted use, distribution, and reproduction in any medium, provided the original work is properly cited.

In this paper, the terminal air defense equipment system of systems (TADESoS) is studied as an example. The TADESoS is an important part of the joint air defense equipment system of systems, which mainly carries out the combat task to the low altitude flight target. The contribution rate evaluation of the TADESoS can provide theoretical basis for guiding the tactical plan of the TADESoS. Aiming at the problems existing in the evaluation of contribution rate of TADESoS, such as the difficulty of describing the structure of system of systems, the strong subjectivity of the evaluation method, and the difficulty of application of the evaluation results, this paper proposes a method of evaluating the contribution rate of the TADESoS based on fault tree. The method describes the structure of the TADESoS by multiattribute nodes. The probability of the top event is calculated by using the probability of the bottom event. Finally, based on the importance of the bottom event, the contribution rate evaluation model of the TADESoS is established, which solves the existing problems in the current research. Finally, the feasibility of the method is verified by an example.

\section{Introduction}

In the modern military struggle, the success or failure of air raid and antiair raid operations directly affects the war situation. In view of the prominent advantages of systematic operation of air raid weapons, if antiair raid weapons do not keep up with the pace of the times, they will be in a passive position in the future war [1]. Therefore, systematic operation of terminal air defense equipment has become an inevitable trend. When terminal air defense equipment is in systematic operation, commanders need to configure the structure of TADESoS, that is, to formulate equipment tactical plan. The rationality of equipment tactical plan directly affects the combat effectiveness of TADESoS. It is difficult to reflect the structural defects of the equipment system of systems by the traditional evaluation method of the rationality of the tactical scheme only relying on the superposition of the tactical and technical indexes. Therefore, a new evaluation method is urgently needed to provide a theoretical basis for guiding the tactical scheme formulation of the TADESoS [2].
The evaluation process describes the contribution of different types of equipment to the system of systems in terms of structural invulnerability; the evaluation results reflect the contribution of the type of equipment to the equipment system of systems in the form of contribution rate evaluation index parameter value and can directly reflect whether there are structural defects in the equipment system of systems. Therefore, it is of great significance to evaluate the contribution rate of TADESoS to guide the formulation of tactical plan of TADESoS.

At present, there are few research results on the evaluation method of structure of equipment system of systems contribution rate, mainly including invulnerability analysis, analytic hierarchy process, and supernetwork method. Based on the analysis of network invulnerability, literature [3] developed the equipment contribution evaluation method and implemented the contribution evaluation for the US missile defense system. In literatures $[4,5]$, the contribution rate evaluation model of equipment system of systems was constructed by using analytic hierarchy process, and the contribution rate evaluation of US air attack equipment system and Russian helicopter equipment system was 
carried out. A supernetwork model for evaluating the survivability of structure of equipment system of systems is proposed in reference [6]. In the above research results, the main problems are that the architecture is difficult to describe, the evaluation method is subjective, and the evaluation results are difficult to apply.

Aiming at the problems existing in the current research, this paper proposes an evaluation method of TADESoS contribution rate based on fault tree. This method integrates the advanced combat theory of "information fire integration" [7] into the evaluation model. At the same time, aiming at the problem that it is difficult to accurately describe the structure of equipment system of systems, this paper describes the structure of the TADESoS through multiattribute nodes and uses the probability of the bottom event to calculate the probability of the top event. Based on the importance of the bottom event, the contribution rate evaluation model of the structure of the terminal air defense equipment system of systems is established. The feasibility of this method is verified by an example.

\section{Fault Tree Construction Method of Structure of TADESoS}

2.1. Analysis of Structure of TADESoS. Structure of the TADESoS analysis is based on the single equipment (subsystem) in the terminal air defense equipment system [8]. Considering the current situation of terminal air defense equipment system construction, and from bottom to top, the structure of the TADESoS is constructed in the way of single equipment (subsystem) $\longrightarrow$ type equipment (subsystem) $\longrightarrow$ system $\longrightarrow$ system. In order to enhance the readability of the article, "equipment (subsystem)" in this chapter is collectively referred to as "equipment."

The structure of the TADESoS constructed in this paper is an open structure; that is, it is constructed on the basis of the current situation of TADESoS [9]. It is assumed that, in a certain period of time in the future, when the TADESoS needs to be added or deleted due to the change of operational needs, it only needs to make corresponding changes on the existing basis [10]. Therefore, this section only analyzes the current TADESoS.

In the construction of structure of TADESoS, because the research object is a combat equipment system, this chapter does not consider the influence of support equipment system on structure of TADESoS. After summarizing the current situation of terminal air defense equipment system construction, combined with the above discussion, the structure of the terminal air defense equipment system is constructed as shown in Figure 1.

In Figure 1, the system of systems layer is the TADESoS, which is the whole combat equipment for terminal air defense mission. The system layer is mainly composed of information protection system, early warning reconnaissance system, integrated command platform system, and fire strike system, which is a necessary combination for equipment to complete specific independent tasks. The type equipment layer consisting of photoelectric countermeasure type equipment, radar countermeasure type equipment, identification of friend or foe (IFF) confrontation type equipment, low altitude and ultralow altitude early warning radar type equipment, medium and low altitude early warning radar type equipment, medium and high altitude early warning radar type equipment, general command and control type equipment, individual command and control type equipment, portable air defense missile type equipment, ground to air missile type equipment, and anti-aircraft gun type equipment is a collection of equipment with the same type of spectrum. Equipment layer is the description of all kinds of single equipment in TADESoS.

\subsection{Fault Tree Event Description of the Terminal Air Defense} Equipment System. Fault tree analysis (FTA) is a method of system reliability analysis $[11,12]$. By analyzing various factors that cause system failure, the inverted tree logic diagram is established. Based on the logic diagram, the combination of factors causing system failure is established by qualitative analysis, and the main factors causing system failure are established by quantitative analysis, so as to find out the solutions to improve system reliability $[13,14]$.

According to the meaning of system of systems, the system of systems is a system with more complex structure than system. Therefore, it is scientific and reasonable to use fault tree analysis method to analyze the structure of the system of systems fault of terminal air defense equipment.

The fault tree consists of events and logic gates. Events include input events and output events. Input events are the cause of fault tree, and output events are the result of fault tree. One or more reasons generate results after logic judgment of logic gate. Therefore, the events in the fault tree are combined through the logic gates between events to form the event relationship, which can reflect the complex structure of the research object $[15,16]$. The terminal air defense equipment system has a complex hierarchical structure, and the fault tree can directly and effectively analyze the composition structure of the terminal air defense equipment system. In view of this, this chapter uses fault tree to analyze structure of the TADESoS.

According to the terms and symbols in the fault tree, combined with the actual needs of TADESoS fault tree analysis, the terms and symbols of structure of TADESoS fault tree are summarized as shown in Table 1.

To analyze the relationship between fault tree events of terminal air defense equipment system, the following assumptions should be made:

Hypothesis 1: there is independence between events

Hypothesis 2: there are only two logical relations in fault tree (AND gate and OR gate)

Hypothesis 3: the objects described by events are only fault and normal states

For the convenience of research, this chapter defines the following variables:

$n$ is the number of basic events

$u_{i}$ is the state of the basic event, $1 \leq i \leq n, i \in N$

$u$ is a vector containing $u_{1}, u_{2}, \ldots, u_{n}$ 


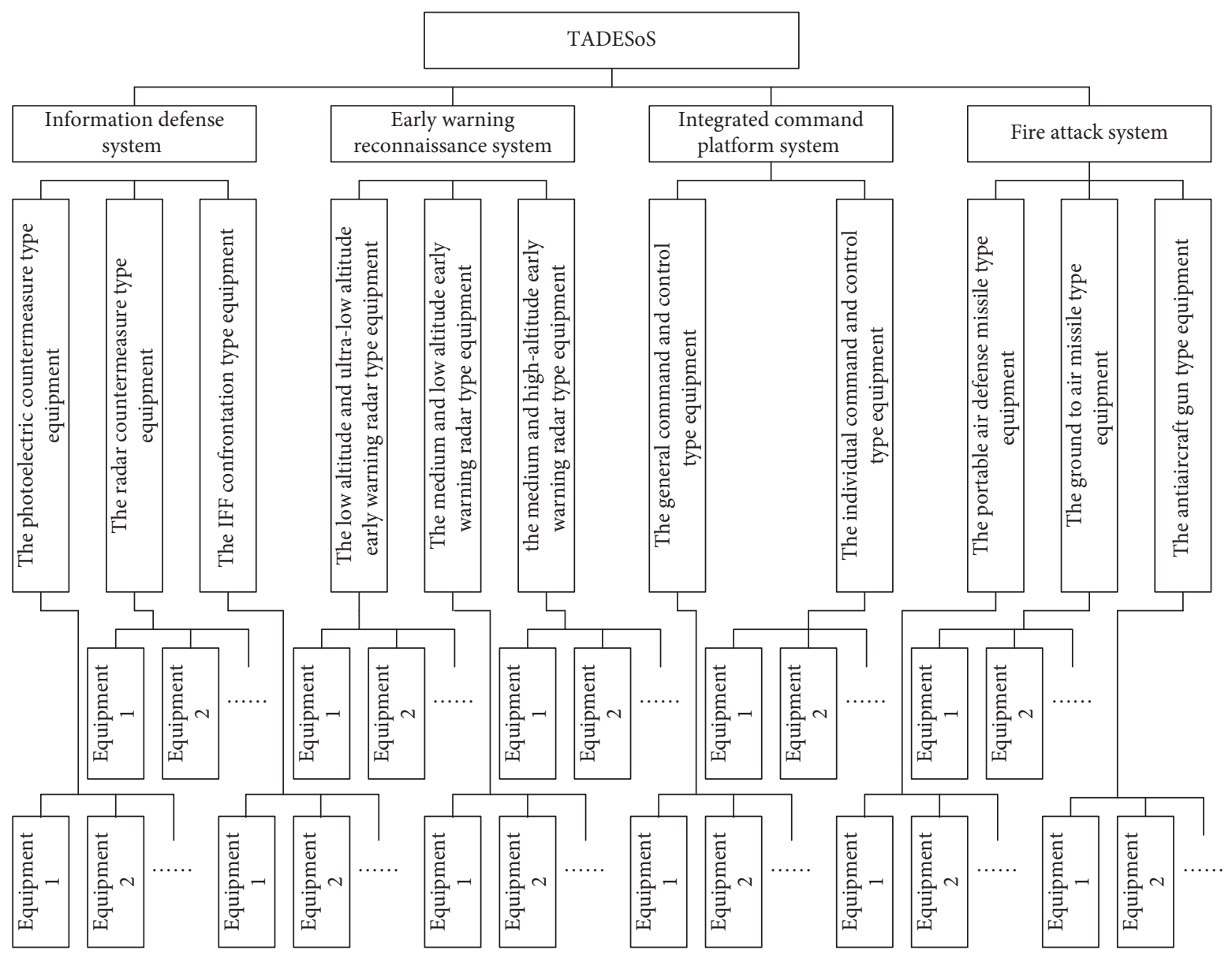

FIGURE 1: Structure of the TADESoS.

TABLE 1: Terms and symbols of fault tree of structure of TADESoS.

\begin{tabular}{|c|c|c|c|}
\hline Category & Name & Symbol & Meaning \\
\hline \multirow{2}{*}{$\begin{array}{l}\text { Logic } \\
\text { gate }\end{array}$} & AND gate & & $\begin{array}{l}\text { Only when all the input events corresponding to an output event occur at the same time can the } \\
\text { output event occur. }\end{array}$ \\
\hline & OR gate & & $\begin{array}{l}\text { Among all the input events corresponding to an output event, the occurrence of any one of the } \\
\text { input events will lead to the occurrence of the output event. }\end{array}$ \\
\hline \multirow{3}{*}{ Events } & Basic events & & $\begin{array}{l}\text { Among all the event, the most basic one that cannot be decomposed is usually located at the } \\
\text { bottom of the fault tree. The basic event is the input event of all events. }\end{array}$ \\
\hline & $\begin{array}{l}\text { Intermediate } \\
\text { events }\end{array}$ & & $\begin{array}{l}\text { The event between the basic event and the top event is the bridge between the qualitative analysis } \\
\text { and quantitative calculation of the logical relationship of events in fault tree. The intermediate } \\
\text { event is not only the output event of the basic event, but also the input event of the upper event. }\end{array}$ \\
\hline & Top events & & $\begin{array}{l}\text { events or intermediate events, is generally located at the highest level of the fault tree. The top } \\
\text { event is the output event of the fault tree. }\end{array}$ \\
\hline
\end{tabular}

$\xi$ represents the state of the top-level event

According to the definition of the above assumption, when an event occurs, the value is " 1 "; when the event does not occur, the value is " 0 ." Therefore, the state of the basic event can be expressed as

$$
u_{i}= \begin{cases}1, & \text { basic events occur, } \\ 0, & \text { basic events do not occur. }\end{cases}
$$

Top-level events can be represented as

$$
\xi= \begin{cases}1, & \text { top level events occur } \\ 0, & \text { top level events do not occur. }\end{cases}
$$

Because the state of all basic events determines the state of top-level events, the state relationship between top-level events and basic events is 


$$
\xi=\xi(u)=\xi\left(u_{1}, u_{2}, \ldots, u_{n}\right) .
$$

$\xi(u)$ is the structure function of TADESoS fault tree, which reflects the composition relationship between basic events and top-level events in TADESoS fault tree.

According to the definition and explanation of OR gate and AND gate in fault tree table of structure of TADESoS, the structure function of OR gate is

$$
\xi(u)=1-\prod_{i=1}^{n}\left(1-u_{i}\right) .
$$

The structure function of AND gate is

$$
\xi(u)=\prod_{i=1}^{n} u_{i} .
$$

2.3. Fault Tree Construction of TADESoS. According to structure of the TADESoS, combined with the fault tree terms and symbol table of structure of the TADESoS, the following variables are defined:

$U_{i}$ is the basic event, $1 \leq i \leq n, i \in N$

$U_{\omega}$ and $U_{\psi}$ represent any basic events, respectively,

$1 \leq \omega, \psi \leq n, \omega, \psi \in N, \omega \neq \psi$

$G$ is the top-level event

$m$ is the number of intermediate events

$M_{j}$ is the intermediate event, $1 \leq j \leq m, j \in N$

$M_{1}$ means information protection system failure

$M_{2}$ means the early warning reconnaissance system failure

$M_{3}$ means the integrated command platform system failure

$M_{4}$ means fire strike system failure

$M_{5}$ means the photoelectric countermeasure equipment failure

$M_{6}$ means the radar countermeasure equipment failure

$M_{7}$ means the IFF confrontation equipment failure

$M_{8}$ means the low altitude and ultralow altitude early warning radar type equipment failure

$M_{9}$ means the medium and low altitude early warning radar type equipment failure

$M_{10}$ means the medium and high altitude early warning radar type equipment failure

$M_{11}$ means the general command and control type equipment failure

$M_{12}$ means the individual command and control type equipment failure

$M_{13}$ means the portable air defense missile type equipment failure

$M_{14}$ means the ground to air missile type equipment failure

$M_{15}$ means the anti-aircraft gun type equipment failure
According to the composition of structure of the TADESoS, the top event in the TADESoS fault tree event represents the TADESoS fault [17]. The basic event is composed of all single equipment failure events in the TADESoS. In addition to the top-level events and basic events, the other events are intermediate events.

According to the above definition of events in TADESoS, combined with the cooperation relationship between different equipment, systems, and system of systems in function and capability, the relationship between events and events in TADESoS is analyzed as follows.

2.3.1. Information Protection System Failure. When one or two types of equipment fail, such as photoelectric countermeasure equipment, radar countermeasure equipment, and IFF countermeasure equipment, the information protection system will have a "short board" of information defense capability due to the lack of some functions, which is very likely to lead to the failure of information protection system. Therefore, the failure events of optoelectronic countermeasure type equipment, radar countermeasure type equipment, IFF countermeasure type equipment, and information protection system constitute OR logic relationship [18].

\subsubsection{Early Warning Reconnaissance System Failure.} When there is one or two types of low altitude and ultralow altitude early warning radar type equipment, medium and low altitude early warning radar type equipment, and medium and high altitude early warning radar type equipment fail, due to the "loopholes" in the early warning airspace, the enemy air attack weapons are very likely to use the "loopholes" in the early warning airspace to successfully implement penetration, and as a result, the early warning reconnaissance system cannot achieve the purpose of early warning [19]. Therefore, it can be considered that the early warning reconnaissance system fails; that is, the early warning reconnaissance system fails. In view of this, the failure events of low altitude and ultralow altitude early warning radar, medium and low altitude early warning radar, medium and high altitude early warning radar, and early warning reconnaissance system constitute OR logic relationship.

\subsubsection{Integrated Command Platform System Failure.} When one of the general command and control type equipment or individual command and control type equipment fails, it is impossible for the decision-maker to command and control the combat unit. Therefore, the occurrence of general command and control type equipment failure event, individual command and control type equipment failure event, and integrated command platform system failure event constitute OR gate logic relationship.

2.3.4. Fire Strike System Failure. In the fire strike system, our terminal air defense cannot complete the firepower alert on the way of marching when the equipment of the portable air 
defense missile type fails. When the ground to air missile type equipment fails, our terminal air defense equipment system cannot complete the fire strike task to the medium and low altitude targets. When the subsystem of anti-aircraft gun type equipment fails, our terminal air defense equipment system cannot complete the fire strike task of ultralow altitude penetration target. Therefore, when one or two types of equipment fail, it will form "loopholes" in the air fire network, which greatly increases the penetration probability of enemy air attack weapons. In view of this, there is OR logic relationship between the fault event of the ground to air missile type equipment, the fault event of the anti-aircraft gun type equipment, and the fault event of the fire strike system.

2.3.5. A Type of Equipment Failure. When a type of equipment is used in practice, it may contain one or more sets of this type of equipment. For example, when the portable air defense missile type equipment undertakes the air fire alert during the March, a March echelon must use two sets of guidance equipment. Therefore, the guidance type equipment consists of two sets of portable air defense missile equipment. When the number of equipment contained in a type of equipment is equal to one, a single equipment event replaces the corresponding type of equipment event. When the number of equipment contained in a type of equipment is greater than one, as long as all the equipment failure events in the type of equipment do not occur, the function or capability of the type of equipment is weakened but still exists. Therefore, only when all the equipment failure events in the type of equipment occur, the corresponding type of equipment failure events will occur. In view of this, the occurrence of a single equipment failure event and the corresponding type of equipment failure event constitute AND gate logical relationship.

\subsubsection{Terminal Air Defense Equipment System Failure.} When any system of information protection system, early warning reconnaissance system, integrated command platform system, and fire strike system fails, the terminal air defense equipment system will show "loopholes" due to the lack of capability attributes, resulting in the loss of system capability. In view of this, the information protection system fault event, early warning reconnaissance system fault event, integrated command platform system fault event, fire strike system fault event, and terminal air defense equipment system fault event constitute OR gate logic relationship.

According to the above analysis content, combined with the fault tree construction method proposed by Bell Laboratories, the fault tree of TADESoS is constructed, as shown in Figure 2.

\section{Value Evaluation Model of Structure of the TADESoS}

The TADESoS is an advanced equipment system of systems in our air defense forces. It has certain information operational capability and firepower strike capability, that is, the ability of information fire integration attack. According to the mission and task characteristics of the terminal air defense equipment system, the terminal air defense equipment system mainly focuses on defense. Therefore, the theory of the "information fire integration" attack is applied to the operation of the TADESoS, which can also be called information defense capability and fire defense capability, that is, the "information fire integration" defense capability.

The traditional Observation-Oriented-Decision-Act (OODA) combat chain model only analyzes the importance of different nodes in the equipment system of systems from the perspective of single chain [20]. With the proposal and practical application of "information fire integration" attack theory, the applicability of single chain model is limited. Therefore, this paper proposes a combat chain model with double chain structure, which can solve the shortcomings of single chain structure and has a certain feasibility and credibility to evaluate the importance of equipment in TADESoS with "information fire integration" defense capability.

The "information fire integration" Defense Dual combat chain (Scout command fire target, information command information target; SCFT, ICIT) of the TADESoS is a dual chain model with common nodes. The dual chain model is composed of information defense operation chain and firepower defense operation chain, which contains two closed loops. For the convenience of research, the following variables and nodes are defined:

Information protection attribute node $I$ : it mainly provides information security protection for our TADESoS, blocks the enemy's intelligence collection of our terminal air defense, and has the ability of electronic information reconnaissance and information jamming.

Early warning and reconnaissance attribute node $S$ : it mainly collects air intelligence to provide intelligence support for the command and decision-making of TADESoS operations.

Command and control attribute node $C$ : it is the nerve center of TADESoS, which mainly analyzes the battlefield situation and issues operational orders. In order to distinguish the amount of information contained in the command and control attribute node in the dual chain model, the command and control attribute node in the fire defense operation chain model is represented as $C_{F}$, and the command and control attribute node in the information defense operation chain model is represented as $C_{I}$.

Fire protection attribute node $F$ : it mainly destroys the combat target by hard kill to prevent the enemy from attacking the defense target of TADESoS.

Target attribute node $T$ : the combat object of our TADESoS.

There are functional associations between different attribute nodes. The connection between the two attribute nodes is defined as transfer module, and the transfer module 


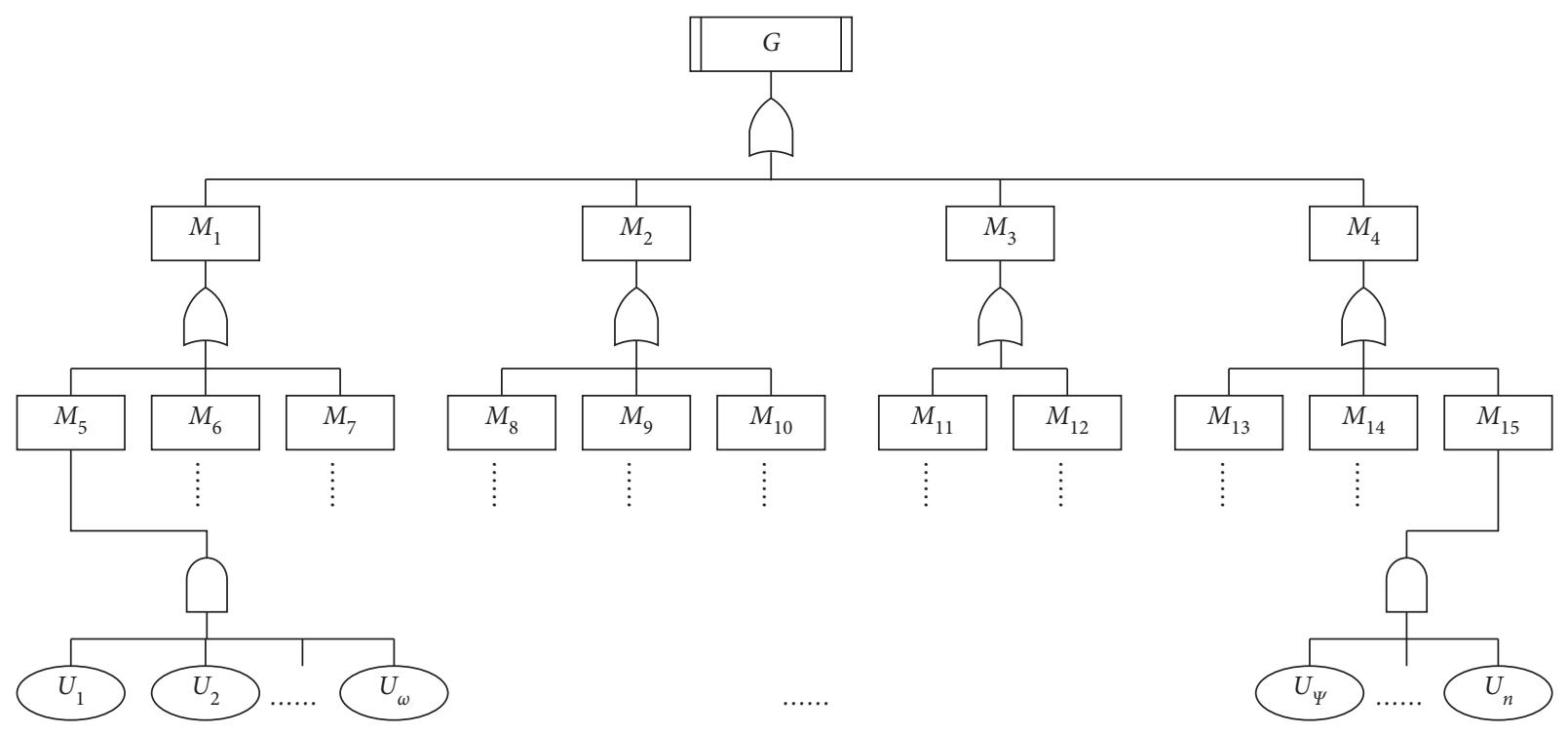

Figure 2: The fault tree of TADESoS.

shown in Table 2 exists in the terminal air defense equipment system. In the table, "-" indicates that there is no connection relationship between the two attribute nodes.

Through the above analysis and discussion, the model diagram of "information fire integration" Defense Dual combat chain of TADESoS is shown in Figure 3.

In Figure 3, S-C-F-T-S is the closed loop of fire defense operation chain, and I-C-I-T-I is the closed loop of information defense operation chain. Fire defense operation chain and information defense operation chain share nodes $\mathrm{C}, \mathrm{F}$, and $\mathrm{T}$. The direction of the arrow indicates the business process order of the node.

The more the number of combat chains of TADESoS is, the less likely the system will be disintegrated by the enemy in the process of operation, that is, the stronger the survivability. Therefore, the number of combat chains of TADESoS can be used as an important index to measure the survivability of the equipment system of systems.

In order to construct the calculation model of combat chain quantity of TADESoS, the following variables are defined:

$\alpha, \beta$ represent any attribute node in the "information fire integrated" Defense Dual combat chain model of TADESoS, $\alpha \neq \beta$

$\mathrm{TM}_{\alpha \beta}$ is the transition matrix between attribute nodes $\alpha$ and $\beta$

$\mathrm{RM}_{\alpha \alpha}$ is the regression matrix of attribute node $\alpha$ in a kill chain loop

$\tau, \phi$ represents any element in attribute nodes $\alpha$ and $\beta$, respectively

$\mathrm{NKC}_{T S C_{F} F T}^{F}$ is the number of fire defense kill chains in TADESoS

$\mathrm{NKC}_{T I C_{I} I T}^{I}$ is the number of information defense kill chains in TADESoS

NKC is the number of effective kill chains of TADESoS $\gamma$ refers to any equipment in the TADESoS

$\mathrm{NKC}_{\gamma}$ is the number of effective kill chains of TADESoS after deleting equipment $\gamma$

$\Delta \mathrm{NKC}_{\gamma}$ is the number of effective kill chains contained in equipment $\gamma$ in TADESoS

$\mathrm{PBA}_{\gamma}$ indicates the possibility that $\gamma$ equipment will be attacked by the enemy

$\mathrm{BEF}_{\gamma}$ is the probability of equipment $\gamma$ failure event

$\mathrm{EFP}_{i}$ is the basic event occurrence probability corresponding to the equipment failure event occurrence probability of $\gamma$

Definition 1. Transition matrix refers to the two-dimensional array of the connection relationship (edge) between adjacent nodes.

Definition 2. Regression matrix refers to the transition matrix formed by a node and the next node in the closed loop of the kill chain. The transition matrix formed by all transfer modules in the closed loop of the kill chain transfers according to the direction order of the closed loop and finally returns to the original position of the node.

Assuming that attribute node $\alpha$ and attribute node $\beta$ constitute a transfer module, the transfer matrix of attribute node $\alpha$ and attribute node $\beta$ is

$$
\mathrm{TM}_{\alpha \beta}=\left[\begin{array}{ccccc}
e_{11} & e_{12} & \cdots & e_{1 \tau} & \cdots \\
e_{21} & e_{22} & \cdots & e_{2 \tau} & \cdots \\
\vdots & \vdots & \cdots & \vdots & \cdots \\
e_{\varphi 1} & e_{\varphi 2} & \cdots & e_{\varphi \tau} & \vdots \\
\vdots & \vdots & \cdots & \vdots & \cdots
\end{array}\right] .
$$

In the transition matrix, the value of $e_{\varphi \tau}$ is 
TABLE 2: TADESoS transfer module.

\begin{tabular}{|c|c|c|c|c|c|}
\hline Node attribute & Attribute node $I$ & Attribute node $S$ & Attribute node $C$ & Attribute node $F$ & Attribute node $T$ \\
\hline Attribute node $I$ & $I \longrightarrow I$ & - & $I \longrightarrow C$ & - & - \\
\hline Attribute node $S$ & - & $S \longrightarrow S$ & $S \longrightarrow C$ & - & - \\
\hline Attribute node $C$ & $C \longrightarrow I$ & - & $C \longrightarrow C$ & $C \longrightarrow F$ & - \\
\hline Attribute node $F$ & - & - & - & - & $F \longrightarrow T$ \\
\hline Attribute node $T$ & $T \longrightarrow I$ & $T \longrightarrow S$ & - & - & - \\
\hline
\end{tabular}

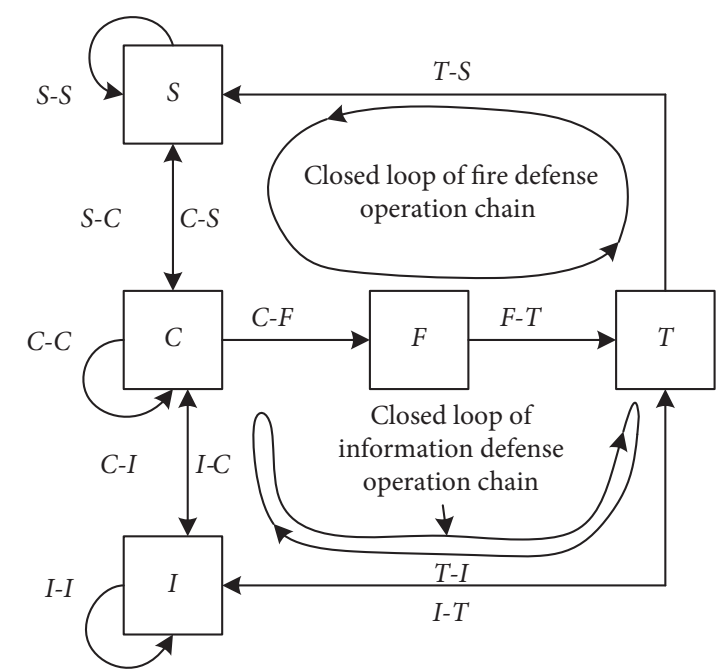

FIGURE 3: Model diagram of "information fire integration" Defense Dual combat chain of TADESoS.

$e_{\phi \tau}= \begin{cases}1, & \text { there is an edge between element } \tau \text { and element } \phi, \\ 0, & \text { there is no edge between element } \tau \text { and element } \phi .\end{cases}$

After the target attribute node is continuously transferred through the transition matrix, it finally returns to the target attribute node and forms a closed loop. According to the closed loop of fire defense operation chain in Figure 3, the path of fire defense closed loop of TADESoS is as follows:

$$
\left\{\begin{array}{l}
T \longrightarrow S \longrightarrow C_{F} \longrightarrow F \longrightarrow T \\
T \longrightarrow S \longrightarrow S \longrightarrow C_{F} \longrightarrow F \longrightarrow T \\
T \longrightarrow S \longrightarrow C_{F} \longrightarrow C_{F} \longrightarrow F \longrightarrow T \\
T \longrightarrow S \longrightarrow C_{F} \longrightarrow C_{F} \longrightarrow F \longrightarrow T
\end{array}\right.
$$

Therefore, the fire defense regression matrix set of TADESoS is

$\mathrm{RM}^{F}=\left\{\mathrm{RM}_{T S C_{F} F T}^{F}, \mathrm{RM}_{T S S C_{F} F T}^{F}, \mathrm{RM}_{T S C_{F} C_{F} F T}^{F}, \mathrm{RM}_{T S S C_{F} C_{F} F T}^{F}\right\}$.

Similarly, the closed loop path of information defense of TADESoS is

$$
\left\{\begin{array}{l}
T \longrightarrow I \longrightarrow C_{I} \longrightarrow I \longrightarrow T, \\
T \longrightarrow I \longrightarrow I \longrightarrow C_{I} \longrightarrow I \longrightarrow T \\
T \longrightarrow I \longrightarrow C_{I} \longrightarrow C_{I} \longrightarrow I \longrightarrow T \\
T \longrightarrow I \longrightarrow I \longrightarrow C_{I} \longrightarrow C_{I} \longrightarrow I \longrightarrow T .
\end{array}\right.
$$

The information defense regression matrix set of TADESoS is

$$
\mathrm{RM}^{I}=\left\{\mathrm{RM}_{T I C_{I} I T}^{I}, \mathrm{RM}_{T I I C_{I} I T}^{I}, \mathrm{RM}_{T I C_{I} C_{I} I T}^{I}, \mathrm{RM}_{T I I C_{I} C_{I} I T}^{I}\right\} .
$$

The number of fire defense kill chains of TADESoS is

$$
\begin{aligned}
\mathrm{NKC}_{T S C_{F} F T}^{F}= & \mathrm{RM}_{T S C_{F} F T}^{F}+\mathrm{RM}_{T S S C_{F} F T}^{F} \\
& +\mathrm{RM}_{T S C_{F} C_{F} F T}^{F}+\mathrm{RM}_{T S S C_{F} C_{F} F T}^{F} .
\end{aligned}
$$

The number of information defense kill chains of TADESoS is

$$
\begin{aligned}
\mathrm{NKC}_{T I C_{I} I T}^{I}= & \mathrm{RM}_{T I C_{I} I T}^{I}+\mathrm{RM}_{T I I C_{I} I T}^{I} \\
& +\mathrm{RM}_{T I C_{I} C_{I} I T}^{I}+\mathrm{RM}_{T I I C_{I} C_{I} I T}^{I}
\end{aligned}
$$

Taking $\mathrm{RM}_{T S C_{F} F T}^{F}$ as an example, the calculation method of the number of kill chains in regression matrix is as follows:

$$
\mathrm{RM}_{T S C_{F} F T}^{F}=\mathrm{TM}_{T S} * \mathrm{TM}_{S C_{F}} * \mathrm{TM}_{C_{F} F} * \mathrm{TM}_{F T}
$$

In the TADESoS of "information fire integration" defense, information defense kill chain and fire defense kill chain cooperate with each other. The set composed of all information defense kill chain and fire defense kill chain is abstracted as nodes, respectively, and their kill chain is abstracted as elements of nodes. The information defense kill chain and fire defense kill chain form a closed loop ICIT $\longrightarrow$ SCFT $\longrightarrow$ ICIT. Therefore, the number of 
effective kill chains of "information fire integration" Defense Dual combat chain of TADESoS can be solved by regression matrix, and its mathematical model is as follows:

$$
\mathrm{NKC}=\mathrm{TM}_{\mathrm{ICIT}} * \mathrm{TM}_{\mathrm{SCFT}} * \mathrm{TM}_{\mathrm{ICIT}} \text {. }
$$

When calculating the number of kill chains of a certain equipment, after deleting the equipment from the TADESoS, the effective number of kill chains of the TADESoS is calculated. The difference between the effective number of kill chains of the TADESoS before and after deleting the equipment is the effective number of kill chains of the equipment in the system of systems. The calculation method is as follows:

$$
\Delta \mathrm{NKC}_{\gamma}=\mathrm{NKC}-\mathrm{NKC}_{\gamma} .
$$

According to the theory of system of systems attack, when analyzing the attack strategy to our TADESoS from the enemy's point of view, we should selectively attack the equipment with a large number of effective kill chains in our TADESoS, so as to achieve the purpose of destroying the structure of our TADESoS to the greatest extent with the least force. When analyzing the equipment that is easy to be attacked by the enemy in our TADESoS from our perspective, the larger the number of effective kill chains contained in the equipment, the easier it is to be attacked by the enemy. Assuming that the equipment is in a failure state that cannot work normally after being attacked for a certain period of time, the easier the equipment is attacked by the enemy, the higher the probability of failure events, that is, the greater the probability of basic events. Therefore, the number of effective kill chains contained in the equipment can be used as the evaluation standard of the basic event probability.

The following hypotheses should be made for the evaluation of the basic event probability of TADESoS:

Hypothesis 1: the equipment is in fault free state during operation

Hypothesis 2: one enemy missile damages only one set of our equipment

Hypothesis 3: after our equipment is attacked, its function will be invalid immediately

Hypothesis 4: the survivability of equipment is not affected by geographical location

In the case of ignoring the difficulty of different equipment in the TADESoS being attacked, according to the above analysis, the possibility of the equipment $\gamma$ in the TADESoS being attacked by the enemy is as follows:

$$
\mathrm{PBA}_{\gamma}=\frac{\Delta \mathrm{NKC}_{\gamma}}{\sum_{s=1}^{n} \Delta \mathrm{NKC}_{s}} .
$$

Because of the emergence of the system, in general,

$$
\sum_{s=1}^{n} \Delta \mathrm{NKC}_{s}>\mathrm{NKC} \text {. }
$$

Because the single combat time of TADESoS is short, the repairability of equipment in the combat process is not considered. In view of this, the probability of equipment $\gamma$ failure event and the probability of the first basic event $i$ are equal:

$$
\mathrm{EFP}_{i}=\mathrm{BEF}_{\gamma}=\mathrm{PBA}_{\gamma}
$$

\subsection{Top-Level Event Probability Evaluation of TADESoS Fault} Tree. In the fault tree of the structure of the TADESoS, many basic events will be repeated. If the traditional probability event solving method is used to solve the top-level event probability, it is inevitable that the analysis process is complex, and the calculation process is huge, which leads to the increase of error rate of calculation results. However, using the minimal cut set to solve the probability of top-level events in the fault tree can effectively avoid the repetition of basic events, thus avoiding the occurrence of the above problems. Therefore, this paper uses the minimal cut set method to solve the probability of top-level events. For the convenience of research, this chapter defines the following variables:

$$
\begin{aligned}
& \frac{q_{k}}{q_{k}} \text { is the } k \text { th minimal cut set } \\
& h \text { is the number of minimal cut sets, } h \in N \\
& R\left(q_{k}\right) \text { is the probability of minimal cut set } q_{k} \\
& \theta \text { is the number of basic events in the minimal cut set } \\
& q_{k}, \theta \in N \\
& R(G) \text { is the probability of top-level events }
\end{aligned}
$$

The occurrence of all the basic events in the minimal cut set will lead to the occurrence of the minimal cut set. Therefore, the probability of the occurrence of the basic events in the minimum cut set and the probability of the occurrence of the minimum cut set constitute an AND gate relationship. According to the mathematical model of and AND gate, the probability of minimum cut set is

$$
R\left(q_{k}\right)=R\left(u_{1} \cap u_{2} \cdots \cap u_{\theta}\right)=\prod_{i=1}^{\theta} \mathrm{EFP}_{i}
$$

The probability of top-level event occurrence in fault tree is determined by the probability of all minimum cut sets. According to the nature and connotation of minimum cut sets, the expression of probability relationship between toplevel event occurrence probability and minimum cut sets is

$$
R(G)=R\left(q_{1} \cup q_{2} \cdots \cup q_{h}\right) .
$$

The basic events in the fault tree of TADESoS can be composed of multiple minimum cut sets, and there are two cases of intersection and disjoint between different minimum cut sets. Therefore, the probability of top-level events should be calculated separately. 
3.1.1. Disjoint between Minimal Cut Sets. When the minimum cut sets do not intersect, the probability of top-level events is the sum of the probability of the minimum cut sets. The probability of top-level events is

$$
R(G)=\sum_{k=1}^{h} R\left(q_{k}\right)
$$

3.1.2. Intersection between Minimal Cut Sets. When the minimum cut sets intersect, because the minimum cut sets contain one or more basic events, the probability of top-level events is not equal to the sum of the minimum cut set probabilities:

$$
R(G) \neq \sum_{k=1}^{h} R\left(q_{k}\right) .
$$

In this case, the disjoint sum expansion method can be used. The disjoint sum expansion method transforms all basic events in the minimal cut set into incompatible events. Suppose that the top-level event contains two minimum cut sets $q_{1}$ and $q_{2}$. Since the minimum cut sets $q_{1}$ and $q_{2}$ intersect, then the complement set $\overline{q_{2}}$ of $q_{1}$ and $q_{2}$ must not intersect. Therefore, the top-level event is

$$
q_{1} \cup q_{2}=q_{1}+\overline{q_{1}} q_{2} .
$$

Therefore, the probability of top-level events of minimum cut sets $q_{1}$ and $q_{2}$ is

$$
R(G)=R\left(q_{1}\right)+R\left(\overline{q_{1}} q_{2}\right) .
$$

Suppose that the top-level event contains three minimum cut sets $q_{1}, q_{2}$ and $q_{3}$, and they intersect each other. According to the above derivation process, the top-level event is

$$
q_{1} \cup q_{2} \cup q_{3}=q_{1}+\overline{q_{1}}\left(q_{2} \cup q_{3}\right)=q_{1}+\left(\overline{q_{1}} q_{2} \cup \overline{q_{1}} q_{3}\right)
$$

It can be deduced from formula (26) that

$$
\begin{aligned}
q_{1} \cup q_{2} \cup q_{3}= & q_{1}+\overline{q_{1}} q_{2}+\overline{\overline{q_{1}} q_{2}} \overline{q_{1}} q_{3}=q_{1}+\overline{q_{1}} q_{2} \\
& +\left(\overline{q_{1}} \cup q_{2}\right)\left(\overline{q_{1}} q_{3}\right) .
\end{aligned}
$$

Further, derived from formula (27),

$$
q_{1} \cup q_{2} \cup q_{3}=q_{1}+\overline{q_{1}} q_{2}+\overline{q_{1} q_{2}} q_{3} .
$$

Therefore, the probability of top-level events of minimum cut sets $q_{1}, q_{2}$, and $q_{3}$ is

$$
R(G)=R\left(q_{1}\right)+R\left(\overline{q_{1}} q_{2}\right)+R\left(\overline{q_{1} q_{2}} q_{3}\right) .
$$

According to the above derivation method, when the top-level event contains $h$ minimum cut sets, and the minimum cut sets intersect, the probability of top-level event occurrence can be expressed as

$$
\begin{aligned}
R(G)= & R\left(q_{1}\right)+R\left(\overline{q_{1}} q_{2}\right)+R\left(\overline{q_{1} q_{2}} q_{3}\right) \\
& +\cdots+R\left(q_{h} \prod_{k=1}^{h-1} \overline{q_{k}}\right), \quad 2 \leq h, h \in N .
\end{aligned}
$$

Therefore, the probability of top-level events is

$$
\begin{aligned}
R(G)= & \sum_{k=1}^{h} R\left(q_{k}\right)-\sum_{k<b=2}^{h} R\left(q_{k} q_{b}\right) \\
& +\sum_{k<b<d=3}^{h} R\left(q_{k} q_{b} q_{d}\right)+\cdots+(-1) R\left(q_{1}, q_{2}, \ldots, q_{h}\right) .
\end{aligned}
$$

3.2. Evaluation of the Importance of the Basic Event of the Fault Tree of the TADESoS. The probability of top-level events in the fault tree of TADESoS is related not only to the probability of basic events, but also to the composition of different basic events. The probability of occurrence of basic events and the composition relationship between basic events determine the importance of basic events.

The indicators describing the importance of basic events are mainly structural importance, probability importance, and key importance, and their meanings are as follows:

3.2.1. Structural Importance. The structural importance describes the influence of the basic events on the top-level events from the structural perspective in all event system of systems of the fault tree. The importance of structure is only related to the location of basic events in all event systems, and not the probability of occurrence of basic events. Therefore, it is a unitary way to analyze the influence of basic events on top-level events from the perspective of structural importance.

3.2.2. Probability Importance. Probability importance describes the influence of the probability change of the basic event on the probability change of the top-level event. Although probability importance is an index to analyze the relationship between the occurrence probability change of basic event and the probability change of top-level event, the structural relationship of each event in the fault tree has been fully considered in the calculation of the failure probability of the top-level event based on the basic event failure probability. Therefore, probability importance can reflect the influence of basic events on top-level events from the aspects of probability change and fault tree structure.

3.2.3. Key Importance. The key importance describes the influence of the probability change rate of the basic event on the probability change rate of the top-level event. The key importance is the improvement of probability importance, and it describes the relationship between the top-level event and the basic event in the way of change rate. The key importance not only has the function of probability 
importance, but also reflects the difficulty of improving the probability of basic events.

In conclusion, the key importance index can reflect the structural value of basic events in all event systems comprehensively. Therefore, this paper uses the key importance index as the evaluation index of the structure value of the terminal air defense equipment system. For the convenience of research, this chapter defines the following variables:

$\mathrm{PIE}_{i}$ is the probability importance of the basic event

$\mathrm{ECI}_{i}$ is the key importance of the basic event

According to the above description of the probability importance of the basic event, the mathematical model of the probability importance of the basic event is

$$
\mathrm{PIE}_{i}=\frac{\partial R(G)}{\partial\left(\mathrm{BEF}_{i}\right)}
$$

According to the meaning of the key importance degree and the mathematical model of the probability importance of the basic event, the mathematical model of the critical importance of the basic event is derived as follows:

$$
\mathrm{ECI}_{i}=\frac{\mathrm{BEF}_{i}}{R(G)} \cdot \mathrm{PIE}_{i}=\frac{\mathrm{BEF}_{i}}{R(G)} \cdot \frac{\partial R(G)}{\partial\left(\mathrm{BEF}_{i}\right)} .
$$

\section{Contribution Rate Evaluation Model of TADESoS Based on Fault Tree}

According to the discussion on the evaluation method of contribution rate of TADESoS in the evaluation theory of contribution rate of TADESoS, the contribution rate evaluation of TADESoS is carried out from the structural dimension. After evaluating the attribute value of the structure of TADESoS, the contribution rate of TADESoS can be evaluated. For the convenience of research, the following variables are defined:

$$
\begin{aligned}
& \eta \text { means any type of equipment } \\
& \mu \text { represents the number of individual equipment } \\
& \text { contained in type } \eta \text { equipment } \\
& \text { con_SC } \mathrm{S}_{v} \text { is the system contribution rate of item } v \text { of } \\
& \text { type } \eta \text { equipment } \\
& \text { con_SC } \eta \text { is the system contribution rate of type } \eta \\
& \text { equipment }
\end{aligned}
$$

The system contribution rate of item $v$ of type $\eta$ equipment is

$$
\text { con_SC } v=\frac{\sum_{i=1}^{n} \mathrm{ECI}_{i}-\left(\sum_{i=1}^{n} \mathrm{ECI}_{i}-\mathrm{ECI}_{v}\right)}{\sum_{i=1}^{n} \mathrm{ECI}_{i}}=\frac{\mathrm{ECI}_{v}}{\sum_{i=1}^{n} \mathrm{ECI}_{i}} .
$$

Therefore, the system contribution rate of type $\eta$ equipment is

$$
\text { con_SC } \text { SC }_{\eta}=\sum_{v=1}^{\mu} \text { con_SC }{ }_{v} \text {. }
$$

According to formulae (34) and (35), the contribution rate evaluation model of TADESoS based on fault tree is

$$
\text { con_SC } \mathrm{SC}_{\eta}=\frac{\sum_{v=1}^{\mu} \mathrm{ECI}_{v}}{\sum_{i=1}^{n} \mathrm{ECI}_{i}} .
$$

\section{Case Analysis}

5.1. Operational Background Scenario of TADESoS. In this military activity, terminal air defense mainly carries out terminal air defense and antimissile task, and the cover target is the front command post of our army. The air attack weapons that the enemy may use and our terminal air defense equipment are shown in Table 3.

According to the possible attack direction of the enemy's air attack weapons, our terminal air defense is equipped with a set of group command and control equipment. The group carries out combat tasks under the command and control of the brigade command organization, and the brigade command organization is equipped with a set of group command and control equipment.

The early warning radar equipment in the early warning reconnaissance system is set at the best predetermined location according to the geographical location of the operation. The ground to air missile equipment and anti-aircraft gun equipment are, respectively, equipped in the operation group, and the operation is directed by the group command post. The equipment in the information protection system is flexibly equipped according to the position of the battle group, early warning radar, and brigade command post. The portable air defense missile equipment is equipped in the early warning radar position and the information protection equipment position, which are difficult to be covered by our group fire.

According to the functional relationship between the equipment in the TADESoS, the operational schematic diagram of the TADESoS is shown in Figure 4 under this operational background.

5.2. Contribution Rate Evaluation of the Terminal Air Defense Equipment System Based on Fault Tree. According to the operational schematic diagram of terminal air defense equipment system, combined with the construction method of transition matrix, the transition matrices of terminal air defense equipment system transition module are constructed: 


$$
\begin{aligned}
& \mathrm{TM}_{T S}=\left[\begin{array}{l}
1 \\
1 \\
1 \\
1
\end{array}\right]^{T}, \\
& \mathrm{TM}_{S S}=\left[\begin{array}{llll}
0 & 0 & 0 & 0 \\
0 & 0 & 0 & 0 \\
0 & 0 & 0 & 0 \\
0 & 0 & 0 & 0
\end{array}\right], \\
& \mathrm{TM}_{S C_{F}}=\left[\begin{array}{ll}
1 & 0 \\
1 & 0 \\
1 & 0 \\
1 & 0
\end{array}\right] \text {, } \\
& \mathrm{TM}_{C_{F} F}=\left[\begin{array}{ll}
1 & 0 \\
1 & 0 \\
0 & 1 \\
0 & 1 \\
0 & 1
\end{array}\right]^{T}, \\
& \mathrm{TM}_{F T}=\left[\begin{array}{l}
1 \\
1 \\
1 \\
1 \\
1
\end{array}\right] \text {, } \\
& \mathrm{TM}_{T I}=\left[\begin{array}{l}
0 \\
0 \\
1 \\
1 \\
1
\end{array}\right]^{T}, \\
& \mathrm{TM}_{I I}=\left[\begin{array}{lllll}
0 & 0 & 0 & 0 & 0 \\
0 & 0 & 0 & 0 & 0 \\
0 & 0 & 0 & 1 & 1 \\
0 & 0 & 1 & 0 & 1 \\
0 & 0 & 1 & 1 & 0
\end{array}\right], \\
& \mathrm{TM}_{I C_{I}}=\left[\begin{array}{ll}
0 & 0 \\
0 & 0 \\
1 & 0 \\
1 & 0 \\
1 & 0
\end{array}\right], \\
& \mathrm{TM}_{C_{I} I}=\left[\begin{array}{ll}
1 & 0 \\
1 & 0 \\
1 & 0 \\
1 & 0 \\
1 & 0
\end{array}\right]^{T}, \\
& \mathrm{TM}_{I T}=\left[\begin{array}{l}
1 \\
1 \\
1 \\
1 \\
1
\end{array}\right] \text {, } \\
& \mathrm{TM}_{C_{F} C_{F}}=\left[\begin{array}{ll}
0 & 1 \\
0 & 0
\end{array}\right], \\
& \mathrm{TM}_{C_{I} C_{I}}=\left[\begin{array}{ll}
0 & 1 \\
0 & 0
\end{array}\right] .
\end{aligned}
$$

According to the calculation method of fire defense kill chain number and information defense kill chain number of TADESoS, the numbers of fire defense kill chain and information defense kill chain are calculated as follows:

$$
\begin{aligned}
\mathrm{NKC}_{T S C_{F} F T}^{F} & =20, \\
\mathrm{NKC}_{T I C_{I} I T}^{I} & =45 .
\end{aligned}
$$

According to the calculation model of the number of effective kill chains of terminal air defense equipment system, the number of effective kill chains of terminal air defense equipment system is calculated under the operational background:

$$
\mathrm{NKC}=900 .
$$

After calculating the number of effective kill chains of TADESoS, the number of effective kill chains of different equipment is calculated according to the method described in formula (16). The calculation results are shown in Table 4:

Substituting the data in Table 4 into formulae (17) and (19), the probability of equipment failure events in TADESoS is calculated, as shown in Table 5:

According to the data in Table 5, the probability of basic events in the fault tree is shown in Table 6:

According to the operational background scenario of this chapter, combined with the construction method of structure of TADESoS fault tree, the structure of the TADESoS fault tree is constructed, as shown in Figure 5:

According to the steps of downward method, the cut set of fault tree of terminal air defense equipment system is solved. The minimum cut set is substituted into the top-level event probability evaluation of fault tree of terminal air defense equipment system, and the function between the basic event occurrence probability and the top-level event occurrence probability can be deduced. Then, the function is imported into the basic event importance evaluation model of terminal air defense equipment system fault tree, and combined with the data in Table 6, the critical importance of basic events is calculated, as shown in Table 7:

The critical importance index of basic events is the value index of structure of the TADESoS in this chapter. The critical importance data of basic events in Table 7 is substituted into the contribution rate evaluation model of terminal air defense equipment system based on fault tree, and the contribution rate of TADESoS is calculated as shown in Table 8.

\subsection{Evaluation Result Analysis of TADESoS Contribution Rate} Based on Fault Tree. In order to analyze the evaluation results of the contribution rate of TADESoS, this chapter uses the form of histogram to express the contribution rate of different types of equipment in TADESoS. The histogram of contribution rate of TADESoS is shown in Figure 6:

In Figure 6, the abscissa represents the name of the evaluated type of equipment, where 1 represents the photoelectric countermeasure type equipment; 2 represents radar countermeasure type equipment; 3 represents IFF confrontation type equipment; 4 represents low altitude and ultralow altitude early warning radar type equipment; 5 represents medium and low altitude early warning radar type equipment; 6 represents the mid high altitude early warning radar type equipment; 7 represents general 
Table 3: Deployment of major equipment of both sides.

\begin{tabular}{|c|c|c|c|c|}
\hline Sequence & $\begin{array}{l}\text { Name of } \\
\text { equipment }\end{array}$ & $\begin{array}{l}\text { Number of } \\
\text { equipment }\end{array}$ & System & Type \\
\hline 1 & Photoelectric countermeasure equipment & 2 & Information protection system & $\begin{array}{c}\text { Information protection } \\
\text { attributes }\end{array}$ \\
\hline 2 & The radar countermeasure equipment & 2 & Information protection system & $\begin{array}{c}\text { Information protection } \\
\text { attributes }\end{array}$ \\
\hline 3 & The IFF confrontation equipment & 1 & Information protection system & $\begin{array}{c}\text { Information protection } \\
\text { attributes }\end{array}$ \\
\hline 4 & $\begin{array}{c}\text { The low altitude and ultralow altitude early } \\
\text { warning radar type equipment }\end{array}$ & 2 & $\begin{array}{l}\text { The early warning } \\
\text { reconnaissance system }\end{array}$ & $\begin{array}{l}\text { Early warning } \\
\text { reconnaissance } \\
\text { attributes }\end{array}$ \\
\hline 5 & $\begin{array}{l}\text { The medium and low altitude early warning radar } \\
\text { type equipment }\end{array}$ & 1 & $\begin{array}{l}\text { The early warning } \\
\text { reconnaissance system }\end{array}$ & $\begin{array}{l}\text { Early warning } \\
\text { reconnaissance } \\
\text { attributes }\end{array}$ \\
\hline 6 & $\begin{array}{l}\text { The medium and high altitude early warning } \\
\text { radar type equipment }\end{array}$ & 1 & $\begin{array}{l}\text { The early warning } \\
\text { reconnaissance system }\end{array}$ & $\begin{array}{c}\text { Early warning } \\
\text { reconnaissance } \\
\text { attributes }\end{array}$ \\
\hline 7 & $\begin{array}{c}\text { The general command and control type } \\
\text { equipment }\end{array}$ & 1 & $\begin{array}{l}\text { The integrated command } \\
\text { platform system }\end{array}$ & $\begin{array}{c}\text { Command and control } \\
\text { attributes }\end{array}$ \\
\hline 8 & $\begin{array}{l}\text { The individual command and control type } \\
\text { equipment }\end{array}$ & 1 & $\begin{array}{l}\text { The integrated command } \\
\text { platform system }\end{array}$ & $\begin{array}{c}\text { Command and control } \\
\text { attributes }\end{array}$ \\
\hline 9 & The portable air defense missile type equipment & 2 & Fire strike system & $\begin{array}{l}\text { Fire protection } \\
\text { attributes }\end{array}$ \\
\hline 10 & $\begin{array}{l}\text { The ground to air missile type equipment } \\
\text { subsystem }\end{array}$ & 1 & Fire strike system & $\begin{array}{l}\text { Fire protection } \\
\text { attributes }\end{array}$ \\
\hline 11 & The anti-aircraft gun type equipment & 2 & Fire strike system & $\begin{array}{l}\text { Fire protection } \\
\text { attributes }\end{array}$ \\
\hline 12 & BGM-109 & 1 & Enemy air attack weapons & $\begin{array}{c}\text { Target } \\
\text { attributes }\end{array}$ \\
\hline
\end{tabular}

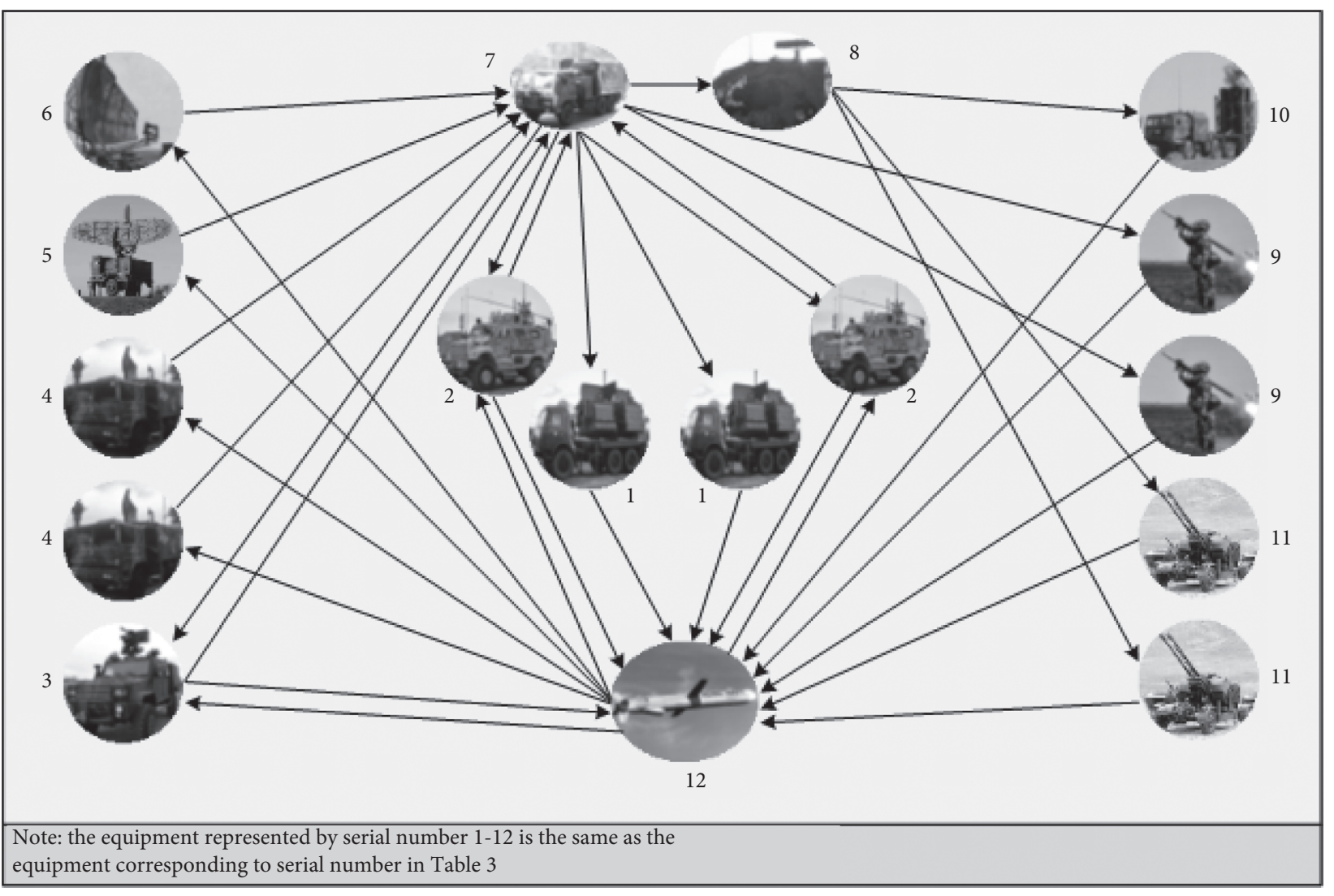

FIgUre 4: Operational diagram of TADESoS. 
TABLE 4: Number of effective kill chains contained in equipment.

\begin{tabular}{ll}
\hline The name of equipment & $\Delta \mathrm{NKC}_{\gamma}$ \\
\hline Photoelectric countermeasure equipment 1 & 180 \\
Photoelectric countermeasure equipment 2 & 180 \\
The radar countermeasure equipment 1 & 580 \\
The radar countermeasure equipment 2 & 580 \\
The IFF confrontation equipment & 580 \\
The low altitude and ultralow altitude early warning radar equipment 1 & 225 \\
The low altitude and ultralow altitude early warning radar equipment 2 & 225 \\
The medium and low altitude early warning radar equipment & 225 \\
The medium and high altitude early warning radar equipment & 900 \\
The general command and control type equipment & 540 \\
The individual command and control equipment & 180 \\
The portable air defense missile equipment 1 & 180 \\
The portable air defense missile equipment 2 & 180 \\
The ground to air missile type equipment subsystem & 180 \\
The anti-aircraft gun equipment 1 & 180 \\
The anti-aircraft gun equipment 2 &
\end{tabular}

TABle 5: Probability of equipment failure events.

\begin{tabular}{lc}
\hline The name of equipment & Probability (\%) \\
\hline Photoelectric countermeasure equipment 1 & 3.3708 \\
Photoelectric countermeasure equipment 2 & 3.3708 \\
The radar countermeasure equipment 1 & 10.8614 \\
The radar countermeasure equipment 2 & 10.8614 \\
The IFF confrontation equipment & 10.8614 \\
The low altitude and ultralow altitude early warning radar equipment 1 & 4.2135 \\
The low altitude and ultralow altitude early warning radar equipment 2 & 4.2135 \\
The medium and low altitude early warning radar equipment & 4.2135 \\
The medium and high altitude early warning radar equipment & 4.2135 \\
The general command and control type equipment & 16.8539 \\
The individual command and control equipment & 10.1124 \\
The portable air defense missile equipment 1 & 3.3708 \\
The portable air defense missile equipment 2 & 3.3708 \\
The ground to air missile type equipment & 3.3708 \\
The anti-aircraft gun equipment 1 & 3.3708 \\
The anti-aircraft gun equipment 2 & 3.3708 \\
\hline
\end{tabular}

TABle 6: Probability of basic events in fault tree.

\begin{tabular}{lc}
\hline Basic events & Probability (\%) \\
\hline$U_{1}$ & 3.3708 \\
$U_{2}$ & 3.3708 \\
$U_{3}$ & 10.8614 \\
$U_{4}$ & 10.8614 \\
$U_{5}$ & 10.8614 \\
$U_{6}$ & 4.2135 \\
$U_{7}$ & 4.2135 \\
$U_{8}$ & 4.2135 \\
$U_{9}$ & 4.2135 \\
$U_{10}$ & 16.8539 \\
$U_{11}$ & 10.1124 \\
$U_{12}$ & 3.3708 \\
$U_{13}$ & 3.3708 \\
$U_{14}$ & 3.3708 \\
$U_{15}$ & 3.3708 \\
$U_{16}$ & 3.3708 \\
\hline
\end{tabular}

command and control type equipment; 8 represents individual command and control type equipment; 9 represents the portable air defense missile type equipment; 10 represents the ground to air missile type equipment; 11 represents anti-aircraft gun type equipment. The ordinate represents the contribution rate parameter value of the evaluated type of equipment.

Through the detailed analysis of Figure 6, the following can be found:

(1) The contribution rate of optoelectronic countermeasure type equipment, low altitude and ultralow altitude early warning radar type equipment, the portable air defense missile equipment, and antiaircraft gun type equipment is low. The system contribution rate of radar countermeasure equipment is low. The contribution rate of middle and low altitude early warning radar type equipment, middle and high altitude early warning radar type 


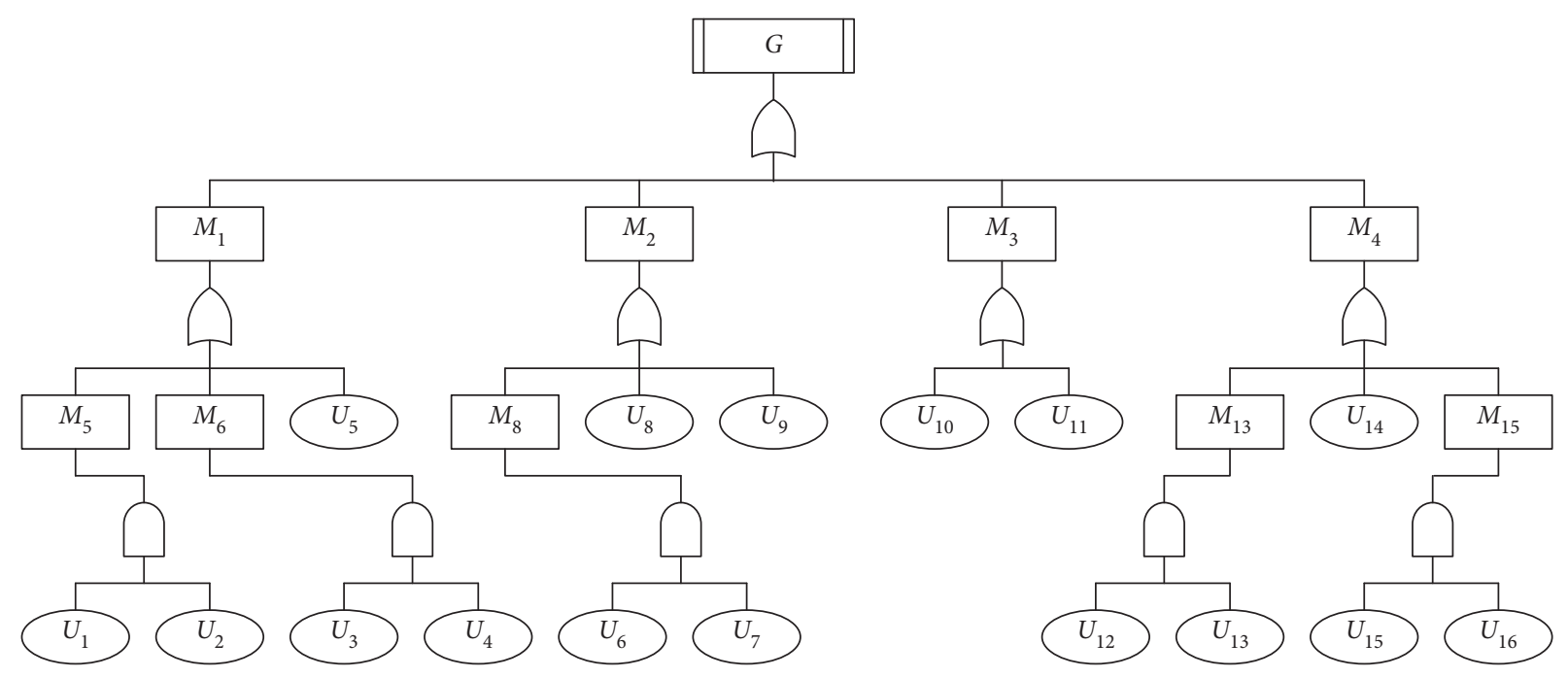

FIgURE 5: Fault tree of structure of the TADESoS.

TABle 7: Critical importance of basic events.

\begin{tabular}{lc}
\hline$U_{i}$ & ECI $_{i}$ \\
\hline$U_{1}$ & 0.0016 \\
$U_{2}$ & 0.0016 \\
$U_{3}$ & 0.0165 \\
$U_{4}$ & 0.0165 \\
$U_{5}$ & 0.1687 \\
$U_{6}$ & 0.0025 \\
$U_{7}$ & 0.0025 \\
$U_{8}$ & 0.0609 \\
$U_{9}$ & 0.0609 \\
$U_{10}$ & 0.2807 \\
$U_{11}$ & 0.1558 \\
$U_{12}$ & 0.0016 \\
$U_{13}$ & 0.0016 \\
$U_{14}$ & 0.0483 \\
$U_{15}$ & 0.0016 \\
$U_{16}$ & 0.0016 \\
\hline
\end{tabular}

equipment, and ground to air missile type equipment is moderate. The contribution rate of IFF confrontation type equipment and group command and control type equipment system is high. The contribution rate of general command and control equipment is high.

(2) The overall distribution of system of systems contribution rate is hierarchical, and there is a large difference between adjacent levels. Therefore, the distribution of system of systems contribution rate in different types of equipment is not balanced.

According to the above rules, combined with the discussion on the contribution rate of TADESoS based on structure in the contribution rate evaluation theory of TADESoS, and in view of the operational background of this case, this chapter gives the following suggestions on the formulation of tactical scheme of TADESoS.:

(1) The system of systems contribution rate of general command and control equipment is high. If the enemy concentrates the superior forces to attack it, after the attack is successful, it will cause serious damage to structure of our TADESoS. Therefore, general command and control type equipment is the primary target of the enemy. When making tactical plans, a set of spare general command and control equipment should be added to improve the survivability of this type of equipment, and on this basis, the general command and control equipment should be deployed in the position with high safety factor.

(2) The contribution rate of IFF confrontation type equipment and general command and control type equipment system is high; therefore, IFF confrontation type equipment and general command and control type equipment are the enemy's key attack targets. However, the general command and control equipment is usually deployed along with the fire equipment, so it is difficult for the enemy to attack this type of equipment. For the location deployment of general command and control type equipment, the combat effectiveness of this type of equipment should be given full play. In order to increase the difficulty of the enemy's attack on the equipment, the location deployment of the equipment should be at a relatively high safety factor.

(3) The contribution rate of middle and low altitude early warning radar type equipment, middle and high altitude early warning radar type equipment, and ground to air missile type equipment is 
TABLE 8: Contribution rate of TADESoS.

\begin{tabular}{lc}
\hline The name of equipment & Contribution rate (\%) \\
\hline Photoelectric countermeasure equipment & 0.3829 \\
The radar countermeasure equipment & 4.0183 \\
The IFF confrontation equipment & 20.5074 \\
The low altitude and ultralow altitude early warning radar equipment & 0.5987 \\
The medium and low altitude early warning radar equipment & 7.4033 \\
The medium and high altitude early warning radar equipment & 7.4033 \\
The general command and control type equipment & 34.1153 \\
The individual command and control equipment & 18.9340 \\
The portable air defense missile equipment & 0.3829 \\
The ground to air missile type equipment & 5.8710 \\
The anti-aircraft gun equipment & 0.3829 \\
\end{tabular}

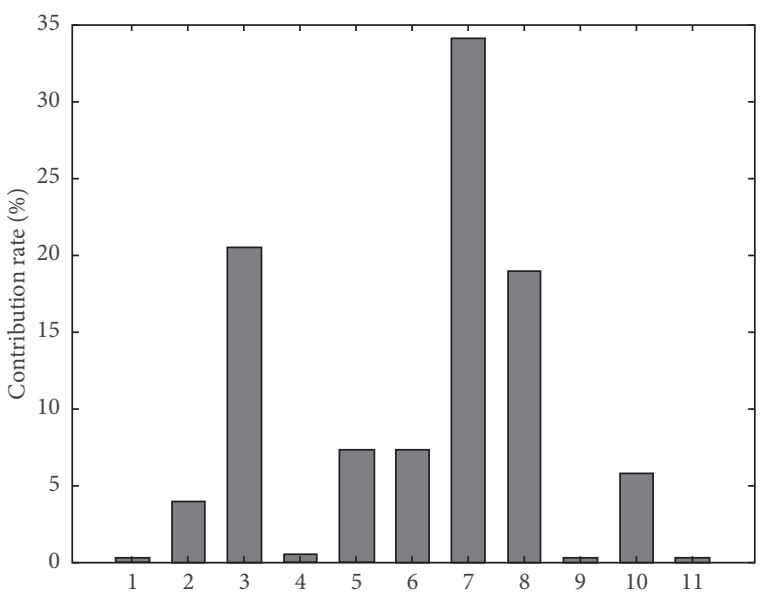

Figure 6: Histogram of contribution rate of TADESoS.

moderate. If the enemy invests a large number of forces to attack them, although it will cause a certain degree of damage to structure of our TADESoS, it will not achieve the effect of system paralysis. For the location deployment of middle and low altitude early warning radar type equipment, middle and high altitude early warning radar type equipment, and ground to air missile type equipment, the safety factor of the geographical location and the influence of the geographical location on the operational efficiency of the above types of equipment should be taken into account.

(4) The contribution rate of optoelectronic countermeasure type equipment, low altitude and ultralow altitude early warning radar type equipment, the portable air defense missile equipment, anti-aircraft gun type equipment, and radar countermeasure type equipment is very low. If the enemy attacks them, even if the attack is successful, the damage to structure of our TADESoS is also very light after the attack is successful. Therefore, if the enemy attacks them, the damage to structure of our TADESoS is very small, and the above types of equipment are not the key targets of enemy attack. In the process of location deployment, we should make full use of the geographical advantages to give full play to its combat effectiveness.

\section{Conclusion}

In this paper, from the perspective of structure of system of systems, a contribution rate evaluation method of TADESoS based on fault tree is proposed. Firstly, the contribution rate evaluation theory of TADESoS is combined with fault tree theory, and the contribution rate evaluation process of terminal air defense equipment system based on fault tree is established. Secondly, combining the fault tree construction principle with structure of the TADESoS, the fault tree construction method of structure of the TADESoS is analyzed. Then, combining the "information fire integration" strike theory with the "system of systems break" theory, the basic event probability evaluation model of TADESoS fault tree is constructed. Based on the basic event probability evaluation model, combined with the top-level event probability evaluation model of fault tree and the basic event importance evaluation model of fault tree, the value evaluation model of structure of the TADESoS is studied. Then, combining the value evaluation model of structure of the TADESoS with the calculation method of contribution rate of structure of the TADESoS, the contribution rate evaluation model of structure of the TADESoS based on fault tree is constructed. Finally, the feasibility of the proposed method is verified by a case study.

The research of this paper does not fully consider the factors such as the probability of spontaneous failure of equipment and the influence of environment on the difficulty of tactical means implementation of both sides. How to integrate the above factors into the contribution rate evaluation of terminal air defense equipment system based on fault tree is the future research direction.

\section{Data Availability}

No data were used to support this study.

\section{Conflicts of Interest}

The authors declare that they have no conflicts of interest.

\section{References}

[1] Q. Yun, B. Song, and Y. Pei, "Modeling the impact of high energy laser weapon on the mission effectiveness of unmanned combat aerial vehicles," IEEE Access, vol. 8, no. 8, pp. 32246-32257, 2020. 
[2] L. X. Chen, "A general equipment system contribution rate evaluation framework," Military Operations Research and Systems Engineering, vol. 34, no. 2, pp. 33-38, 2020.

[3] S. He, K. W. Yang, and J. Liang, "Evaluation of equipment contribution based on network survivability," Fire and Command and Control, vol. 42, no. 8, pp. 87-91, 2017.

[4] G. Z. Bu, "Evaluation method of equipment contribution rate to combat system based on AOE model," Fire and Command Control, vol. 45, no. 12, pp. 18-22, 2020.

[5] B. C. Lou, "The method of evaluating the contribution rate of helicopter system based on analytic hierarchy process," $\mathrm{He}$ licopter Technology, vol. 43, no. 1, pp. 6-10, 2020.

[6] F. X. Luo and L. Zhou, "Complex SOS network model based on hypernetwork," Military Operations Research and Systems Engineering, vol. 31, no. 3, pp. 35-41, 2017.

[7] W. S. Yang, Y. Wang, and Y. Yang, "Effectiveness evaluation of combat network under different node attack strategies based on combat ring," Systems Engineering and Electronic Technology, vol. 12, no. 4, pp. 1789-1794, 2021.

[8] A. F. Kinerber, "Impact of value management on building projects success: structural equation modeling approach," Journal of Construction Engineering and Management, vol. 147, no. 4, pp. 34-56, 2021.

[9] Q. Cao and T. Jing, "Evolutionary game framework of weapon equipment architecture," Military Operations Research and Systems Engineering, vol. 29, no. 1, pp. 50-55, 2015.

[10] X. Wu, T. H. Wang, and Z. H. Gao, "Construction and optimization method of weapon equipment operational test evaluation index system," Fire and Command Control, vol. 45, no. 3, pp. 75-80, 2020.

[11] Y. He, Z. Chen, Y. Zhao, X. Han, and D. Zhou, "Mission reliability evaluation for fuzzy multistate manufacturing system based on an extended stochastic flow network," IEEE Transactions on Reliability, vol. 69, no. 4, pp. 1-15, 2020.

[12] Y. He, Y. Zhao, X. Han, D. Zhou, and W. Wang, "Functional risk-oriented health prognosis approach for intelligent manufacturing systems," Reliability Engineering \& System Safety, vol. 203, no. 3, Article ID 107090, 2020.

[13] L. Zhu, D. Chen, and P. Feng, "Equipment operational reliability evaluation method based on RVM and PCA-Fused features," Mathematical Problems in Engineering, vol. 2021, Article ID 6687248, 9 pages, 2021.

[14] Y. Zhao, C. Lu, C. Fei et al., "Transient reliability evaluation approach of flexible mechanism with GA-extremum neural network," Mathematical Problems in Engineering, vol. 2020, Article ID 6661712, 11 pages, 2020.

[15] Z. Liu, H. Zhang, S. Wang, W. Hong, J. Ma, and Y. He, "Reliability evaluation of public security face recognition system based on continuous bayesian network," Mathematical Problems in Engineering, vol. 2020, Article ID 6287394, 9 pages, 2020.

[16] X. Liu, Z. Liu, J.-T. Chen et al., "Ergonomic reliability assessment for passenger car interface design based on EWMMADM and human cognitive reliability experiments," Mathematical Problems in Engineering, vol. 2020, Article ID 4757202, 10 pages, 2020.

[17] X. J. Yin, X. F. Hu, and M. Rong, "Review and prospect of system contribution evaluation methods," Journal of System Simulation, vol. 31, no. 6, pp. 1027-1038, 2019.

[18] C. K. Luo, Y. X. Chen, and Z. He, "Evaluation method of contribution rate of aviation equipment architecture based on fault tree analysis," Journal of National University of Defense Science and Technology, vol. 43, no. 1, pp. 155-162, 2021.
[19] T. Liu, "Research on evaluation method of tactical data link system contribution rate," Journal of China Academy of Electronic Sciences, vol. 16, no. 2, pp. 189-191, 2021.

[20] L. Wang, B. Li, and Z. A. Qiu, "Contribution rate evaluation of air to air missile weapon system," Aeronautical Weapons, vol. 28, no. 1, pp. 20-25, 2021. 